Review began 09/15/2021 Review ended 09/20/2021 Published 09/23/2021

(c) Copyright 2021

Singh et al. This is an open access article distributed under the terms of the Creative Commons Attribution License CC-BY 4.0., which permits unrestricted use, distribution, and reproduction in any medium, provided the original author and source are credited.

\title{
A Systematic Review of Tafamidis in Patients With Transthyretin Amyloid Cardiomyopathy
}

Bishnu Mohan Singh ${ }^{1,2}$, Narayan Bohara ${ }^{3}$, Kamal Gautam ${ }^{4}$, Madan Basnet ${ }^{5}$, Sistu KC ${ }^{6}$, Binod KC ${ }^{7}$, Anuradha Raut ${ }^{8}$, Abisha Phudong ${ }^{9}$, Jeevan Gautam ${ }^{10}$

1. Division of Clinical and Translational Research, Larkin Community Hospital, Miami, USA 2. Emergency and General Medicine, Patan Academy of Health Sciences, Lalitpur, NPL 3. Cardiology, Oxford University Clinical Research Unit, Patan Academy of Health Sciences, Lalitpur, NPL 4. Internal Medicine, Oxford University Clinical Research Unit, Patan Academy of Health Sciences, Lalitpur, NPL 5. Medicine, Nepalese Army Institute of Health Sciences, Kathmandu, NPL 6. Internal Medicine, Patan Academy of Health Sciences, Lalitpur, NPL 7. Internal Medicine, Upstate University Hospital, New York, USA 8. Internal Medicine, Nepal Medical College and Teaching Hospital, Kathmandu, NPL 9 Emergency Department, Care and Cure Multispeciality Hospital, Lalitpur, NPL 10. Internal Medicine, Tribhuvan University Teaching Hospital, Kathmandu, NPL

Corresponding author: Bishnu Mohan Singh, bishnusingh2047@gmail.com

\section{Abstract}

Transthyretin amyloid cardiomyopathy disease burden is increasing daily due to advancements in diagnostic and imaging modalities in the modern world. Tafamidis is one of many therapeutic options. The main objective of this review is to study the role of Tafamidis in slowing the progression of transthyretin cardiomyopathy (TTR-CM) by analyzing randomized controlled trials (RCTs) and non-RCTs of Tafamidis. We searched for published papers of Tafamidis in the English language in electronic databases like Google Scholar, PubMed, Cochrane Library, and PubMed Central. We imported the resulting articles from our search to Mendeley software. Four reviewers removed the duplicates and performed title and abstract screening of the articles. The same reviewers obtained the full-text of relevant articles and did fulltext screening based on eligibility criteria. Finally, five reviewers performed a quality assessment of RCTs using the Cochrane risk of bias assessment and of non-RCTs by a checklist prepared by Downs and Black. Any disagreements about any process were resolved by a discussion with other authors. One RCT and five non-RCTs of Tafamidis were included in this systematic review. From the non-RCTs, stability was observed in different parameters like echocardiographic findings, cardiac biomarkers, and ECG in patients with transthyretin cardiomyopathy during the study duration with Tafamidis. ATTR-ACT (Tafamidis in Transthyretin Cardiomyopathy Clinical Trial) trial demonstrated reduction of cardiovascular events and allcause mortality in the Tafamidis group in comparison to placebo. In both RCT and non-RCTs, Tafamidis was established as a safe and tolerable drug for patients with TTR-CM. Our study proved the role of Tafamidis in reducing cardiovascular events, all-cause mortality, and the progression of cardiac disease in TTR-CM patients. In addition to five non-RCTs, current evidence is based on the findings of only one RCT of Tafamidis. Hence, evidence from additional RCTs is required to strongly support the stability of parameters like echocardiographic findings, cardiac biomarkers, and ECG with Tafamidis use.

Categories: Cardiac/Thoracic/Vascular Surgery, Cardiology, Internal Medicine

Keywords: randomized controlled trial, cardiomyopathies, amyloid, ttr-stabilizing drug, cardiac biomarkers, echocardiography, outcomes, heart failure, tafamidis, transthyretin amyloid cardiomyopathy

\section{Introduction And Background}

Amyloidosis refers to a group of heterogeneous diseases caused by the extracellular tissue deposition of fibrils composed of abnormally folded proteins. It is a multi-systemic disease affecting various organs, including the heart, kidney, liver, lungs, skin, central and peripheral nervous systems, muscles, skin, etc. Although there are more than 30 proteins causing amyloid deposition in various systems [1], cardiac involvement is mainly caused by misfolded transthyretin (ATTR) or immunoglobulin light chain (AL) depositions [2]. ATTR amyloidosis can occur due to normal wild-type amyloidogenic TTR (transthyretin) protein or due to a variant of the TTR gene (hereditary ATTR amyloidosis, ATTRv). Cardiac involvement can cause diastolic or systolic dysfunction, arrhythmias, heart block, or infarctions due to amyloid deposition in coronary vessels.

Transthyretin amyloid cardiomyopathy is often an under-diagnosed cause of heart failure, especially in the older population [3]. It usually presents with signs and symptoms of heart failure like dyspnea, syncope, fatigue, and orthostatic hypotension. It is a rapidly progressive disease, with a median survival of untreated cases of wild-type ATTR as low as 3.6 years [4]. The mainstay of treatment is symptomatic care of heart failure, which is more beneficial in patients with ATTRm (familial transthyretin-associated) and ATTRwt (wild-type transthyretin-associated) amyloidosis. Surgical treatment options like heart transplantation, liver transplantation to remove mutant TTR from blood may be helpful to some patients [5-6]. Patisiran and Inotersen are RNA-targeted therapies approved by the US Food and Drug Administration (FDA) for transthyretin-mediated hereditary amyloidosis polyneuropathy but are emerging therapies for transthyretin 
cardiomyopathy (TTR-CM) [7]. In contrast, Tafamidis (TTR stabilizer) is an FDA-approved drug for TTR-CM patients. Although a few clinical studies have shown a beneficial effect of Tafamidis in slowing the progression of amyloid cardiomyopathy, a thorough study comparing the overall effects of Tafamidis is still lacking. In this study, we aim to examine the effectiveness of Tafamidis in slowing the progression of cardiomyopathy and its safety profile, effect on various cardiac parameters, and overall improvement of symptoms by evaluating existing clinical studies.

\section{Review \\ Methods}

We followed the guidelines of the Preferred Reporting Items for Systematic reviews and Meta-Analyses (PRISMA 2020) for conducting the systematic review [8].

\section{Study Protocol}

We performed preliminary searches and literature reviews on our research question. We then prepared our protocol according to the guidelines of the Preferred Reporting Items for Systematic Review and MetaAnalysis Protocols 2015 (PRISMA-P 2015) [9]. We published our protocol in the Research Registry, with a unique identifying number: reviewregistry1177.

\section{Search Strategy}

We used electronic databases like PubMed, Cochrane Library, PubMed Central (PMC), and Google Scholar for searching relevant articles to answer our research question until May 2021. We customized our search to include any clinical studies that had evaluated Tafamidis's role in treating transthyretin amyloid cardiomyopathy. We searched for English language studies conducted in human subjects. The search strategies for different electronic databases are shown in Appendices.

\section{Study Selection}

After we completed our search, we imported all articles into Mendeley software. Then, we removed the duplicates in the Mendeley software. Four reviewers (N. B., K.G., B.M.S., and S.K.) independently screened the articles based on their titles and abstracts. The same reviewers again did full-text screening independently. All conflicts were resolved by discussion with other authors. At every step, we used our eligibility criteria to screen and finally selected the studies included in our systematic review. The inclusion criteria were as follows: (i) randomized controlled trials (RCTs), non-RCTs, or single-arm studies of Tafamidis that included patients with transthyretin amyloid cardiomyopathy, either wild-type or varianttype; (ii) studies done on patients of any race and age 18 years or above; (iii) studies with the following outcomes: TTR stabilization, cardiac biomarkers, ECG or Holter parameters, echocardiographic parameters, functional tests, quality of life, arrhythmias, hospital admission, and death; (iv) studies published in the English language. The exclusion criteria were as follows: (i) systematic reviews, meta-analyses, case reports, and case series; (ii) studies done on healthy volunteers only, the pediatric population ( $<18$ years), diseases other than transthyretin amyloid cardiomyopathy; (iii) studies with only non-cardiovascular outcomes; (iv) studies published in a non-English language.

\section{Data Extraction}

We extracted the following data from the studies included in our systematic review: (i) participants in the Tafamidis group and the control group; (ii) duration of follow-up; (iii) participant characteristics like mean age, sex, mean body mass index (BMI), and race; (iv) the dose of Tafamidis; (v) target population; (vi) study design; (vii) adverse events noted among participants; (viii) changes in the level of cardiac biomarkers during the study period; (ix) all-cause mortality; (x) cardiovascular-related hospitalization; (xi) ambulatory changes; and (xii) quality of life assessments during the study duration. Five reviewers (N.B., K.G., M.B., J.G., B.K., and A.R.) extracted the data independently, and any conflicts during the process of data extraction were resolved by discussion with other authors.

\section{Quality Assessment}

We assessed the quality of the included studies by using the Cochrane quality assessment tool for RCTs [10], and a quality assessment of the included non-RCTs was performed using a checklist by Downs and Black [11]. We analyzed the risk of bias in RCT using the risk of bias 2 (RoB 2) tool under the following headings: random sequence generation (selection bias), allocation sequence concealment (selection bias), blinding of participants and personnel (performance bias), blinding of outcome assessment (detection bias), incomplete outcome data (attrition bias), selective outcome reporting (reporting bias), and other potential sources of bias. Depending on the risk of bias, the tool rated RCT as "Low risk," "Unclear risk," and "High risk." Five reviewers (B. M. S., K.G., M.B., N.B., and A.P.) independently assessed the risk of bias of included studies. Any disagreements were resolved by discussion with other authors. 


\section{Cureus}

\section{Results}

Literature Selection

On thorough literature search, we found 691 articles from various databases, of which we found 87 from PubMed, 370 from PMC, 42 from Cochrane Library, and 192 from Google Scholar. Ninety duplicates were found and hence were excluded. On screening the remaining articles, 504 articles were excluded during the title and abstract screening, as they were found to be of the wrong study design (literature reviews or opinion articles). The remaining 97 articles were further evaluated for inclusion in this systematic review by performing full-text screening. Ninety-one articles were excluded, as they did not meet the inclusion criteria during full-text screening. As a result, six studies were included for data extraction, which included one RCT and five non-RCTs. Figure 1 shows the PRISMA flow chart of the studies evaluated in this systematic review.

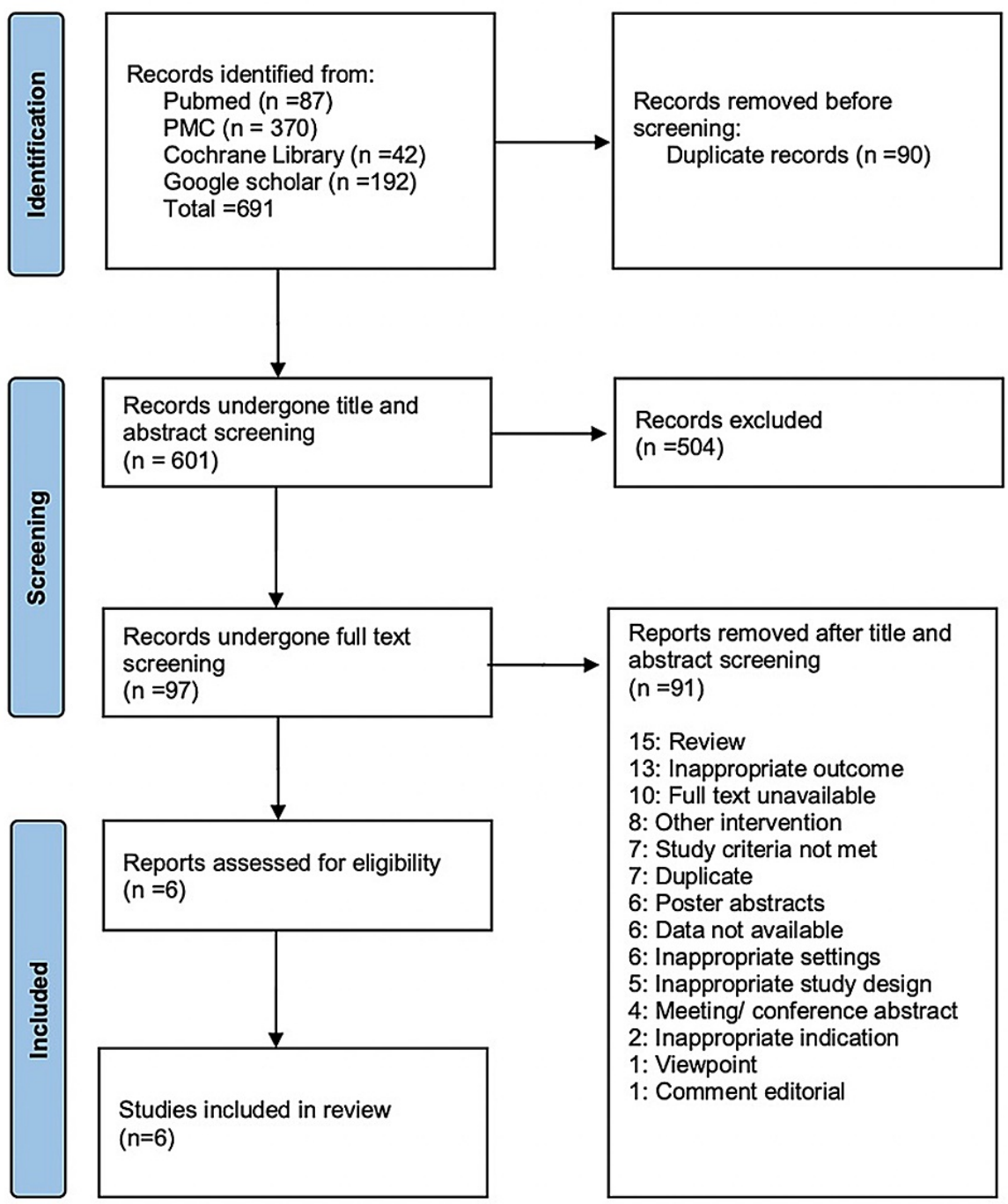

FIGURE 1: PRISMA flow chart of the studies included in the systematic review

PRISMA: Preferred Reporting Items for Systematic Reviews and Meta-Analyses

Included Studies and Quality Assessment

The RCT included in this systematic review is ATTR-ACT (Tafamidis in Transthyretin Cardiomyopathy Clinical Trial) [12]. ATTR-ACT is a phase III multicenter double-blinded study assessing 441 patients of cardiac amyloidosis, 106 with ATTRm (familial transthyretin-associated forms of amyloid) and 335 with ATTRwt (wild-type transthyretin-associated forms of amyloid) [12]. The five non-RCTs included in this systematic review are as follows: (i) Three phase II studies [13-15] assessing the efficacy of Tafamidis in 31, 21, and 21 patients; (ii) one multicenter observational study [16] assessing the efficacy of Tafamidis in 61 


\section{Cureus}

patients; (iii) one phase III study [17] assessing the efficacy of Tafamidis in 10 patients.

Figure 2 represents the risk of bias graph of the ATTR-ACT trial [12] assessed using the Cochrane RoB 2 tool. In the ATTR-ACT trial [12], randomization was carried out appropriately with adequate concealment of treatment allocation. Also, in the ATTR-ACT trial [12], care providers, participants, and outcome assessors were blinded to treatment allocation. However, there was significant missing data in both the Tafamidis and placebo groups. Thirty-four point forty-seven percent (34.47\%) of participants' data was missing in the Tafamidis group due to various reasons while the percentage was 51.98 in the placebo group. Hence, there was much imbalance between the two groups regarding the missing data in the ATTR-ACT trial. Table 1 represents the risk of bias assessment of five non-RCTs using the Downs and Black assessment checklist.

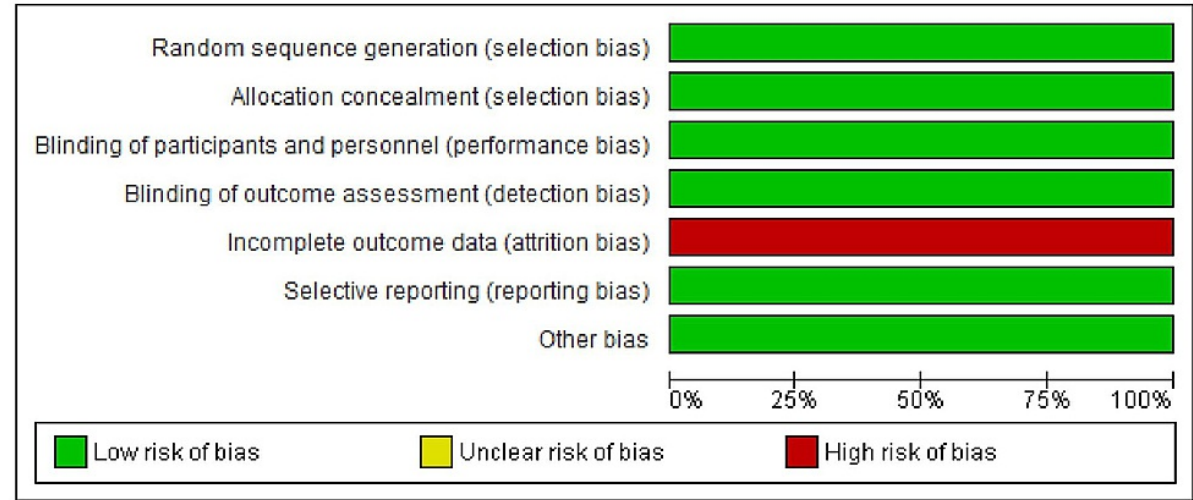

FIGURE 2: Risk of bias graph of ATTR-ACT trial assessed using the Cochrane risk of bias 2 tool

ATTR-ACT: Tafamidis in Transthyretin Cardiomyopathy Clinical Trial

\section{Question no.}

i. Is the hypothesis/aim/objective of the study clearly described?

ii. Are the main outcomes to be measured clearly described in the introduction or methods sections?

iii. Are the characteristics of the patients included in the study clearly described?

iv. Are the interventions of interest clearly described?

v. Are the distributions of principal confounders in each group of patients to be compared clearly described?

vi. Are the main findings of the study clearly described?

vii. Does the study provide estimates of the random variability in the data for the main outcomes?

viii. Have all important adverse events that may be a consequence of the intervention been reported?

ix. Have the characteristics of patients lost to follow-up been described?

x. Have actual probability values been reported (e.g., 0.035 rather than $<0.05$ ) for the main outcomes except where the probability value is less than 0.001 ?

xi. Were all the subjects asked to participate in the study representative of the entire population from which they were recruited?

xii. Were those subjects who were prepared to participate representative of the entire population from which they were recruited?

xiii. Were the staff, places, and facilities where the patients were treated representative of the treatment the majority of patients receive?

\begin{tabular}{|c|c|c|c|c|}
\hline $\begin{array}{l}\text { Maurer } \\
\text { et al. } \\
{[13]}\end{array}$ & $\begin{array}{l}\text { Damy } \\
\text { et al. } \\
{[14]}\end{array}$ & $\begin{array}{l}\text { Merlini } \\
\text { et al. } \\
\text { [15] }\end{array}$ & $\begin{array}{l}\text { Cortese } \\
\text { et al. } \\
{[16]}\end{array}$ & $\begin{array}{l}\text { Yukio } \\
\text { et al. } \\
{[17]}\end{array}$ \\
\hline Yes & Yes & Yes & Yes & Yes \\
\hline Yes & Yes & Yes & No & Yes \\
\hline Yes & Yes & Yes & Yes & Yes \\
\hline Yes & Yes & Yes & No & Yes \\
\hline Yes & Yes & Yes & Yes & Yes \\
\hline Yes & Yes & Yes & No & Yes \\
\hline Yes & Yes & No & Yes & Yes \\
\hline No & Yes & Yes & Yes & Yes \\
\hline Yes & Yes & Yes & Yes & Yes \\
\hline No & No & No & Yes & No \\
\hline No & No & No & Yes & No \\
\hline No & Yes & No & No & No \\
\hline No & No & Yes & Yes & No \\
\hline
\end{tabular}




\section{Cureus}

xiv. Was an attempt made to blind study subjects to the intervention they have received? No

xv. Was an attempt made to blind those measuring the main outcomes of the intervention? No

xvi. If any of the results of the study were based on "data dredging," was this made clear?

xvii. In trials and cohort studies, do the analyses adjust for different lengths of follow-up patients, or in case-cohort studies, is the time period between the intervention and outcome the same for cases and controls?

xviii. Were the statistical tests used to assess the main outcomes appropriate?

xix. Was compliance with the intervention(s) reliable?

$\mathrm{xx}$. Were the main outcome measures used accurate (valid and reliable)?

xxi. Were the patients in different intervention groups (trials and cohort studies), or were the cases and controls (case-control studies) recruited from the same population?

xxii. Were study subjects in different intervention groups (trials and cohort studies), or were the cases and controls (case-control studies) recruited over the same period of time?

xxiii. Were study subjects randomized to intervention groups?

xxiv. Was the randomized intervention assignment concealed from both patients and health care staff until recruitment was complete and irrevocable?

xxv. Was there an adequate adjustment for confounding in the analyses from which the main findings were drawn?

xxvi. Were losses of patients to follow-up taken into account?

$\begin{array}{llll}\text { No } & \text { No } & \text { No } & \text { No } \\ \text { No } & \text { No } & \text { No } & \text { No } \\ \text { Yes } & \text { Yes } & \text { No } & \text { No }\end{array}$

U Yes Yes No No

No Yes No No No

Yes Yes Yes Yes Yes

$\begin{array}{lllll}U & U & U\end{array}$

Yes Yes Yes Yes Yes

NA NA NA NA NA

NA NA NA NA NA

No No No No No

No No No No No

No No No No no

No No No No No

\section{TABLE 1: Risk of bias assessment of non-RCTs using the Downs and Black assessment checklist}

RCT: randomized controlled trial; NA: not applicable; U: unable to determine

Table 2 shows the characteristics of participants of the studies included in the systematic review. The study characteristics of the RCT and non-RCTs included in the review are shown in Table 3. Table 4 shows the changes in echocardiographic parameters, cardiac biomarkers, quality of life, and percentage of TTR (transthyretin) stabilization achieved during the study. 


\section{Cureus}

\begin{tabular}{|c|c|c|c|c|c|c|c|}
\hline \multirow{2}{*}{$\begin{array}{l}\text { Patient } \\
\text { Characteristics }\end{array}$} & \multicolumn{2}{|l|}{ ATTR-ACT trial [12] } & \multirow{2}{*}{$\begin{array}{l}\text { Maurer } \\
\text { et al. } \\
{[13]}\end{array}$} & \multirow{2}{*}{ Damy et al. [14] } & \multirow{2}{*}{ Merlini et al. [15] } & \multirow{2}{*}{$\begin{array}{l}\text { Cortese } \\
\text { et al. } \\
{[16]}\end{array}$} & \multirow{2}{*}{$\begin{array}{l}\text { Yukio } \\
\text { et al. } \\
\text { [17] }\end{array}$} \\
\hline & Tafamidis $(n=264)$ & Placebo $(n=177)$ & & & & & \\
\hline $\begin{array}{l}\text { Age in years, } \\
\text { Mean (SD) }\end{array}$ & $74.5(7.2)$ & $74.1(6.7)$ & $\begin{array}{l}76.7 \\
(68.7- \\
86.5)^{\star}\end{array}$ & $63.1(9.9)$ & $63.1(9.9)$ & $62(11)$ & $\begin{array}{l}60.1 \\
(13)\end{array}$ \\
\hline $\begin{array}{l}\text { Sex, Male No. } \\
(\%)\end{array}$ & $241(91.3)$ & $157(88.7)$ & $\begin{array}{l}29 \\
(93.5)\end{array}$ & $13(61.9)$ & $13(61.9)$ & $42(69)$ & $7(70)$ \\
\hline Race No. (\%) & $\begin{array}{l}\text { White- } 211 \text { (79.9); } \\
\text { Black- } 37 \text { (14.0); } \\
\text { Asian- } 13(4.9) ; \\
\text { Other- } 3 \text { (1.1) }\end{array}$ & $\begin{array}{l}\text { White-146 (82.5); } \\
\text { Black- } 26 \text { (14.7); } \\
\text { Asian- } 5 \text { (2.8); } \\
\text { Other- } 0\end{array}$ & N/A & $\begin{array}{l}\text { Afro-Caribbean- } 1 \\
\text { (4.8); Asian- } 1 \\
(4.8) ; \text { Caucasian- } \\
19(90.5)\end{array}$ & $\mathrm{N} / \mathrm{A}$ & N/A & $\mathrm{N} / \mathrm{A}$ \\
\hline $\begin{array}{l}\text { Body mass } \\
\text { index Mean } \\
\text { (SD) }\end{array}$ & NA & NA & NA & NA & $\begin{array}{l}\mathrm{mBMI}=1,052.5(206.7) \\
\mathrm{kg} / \mathrm{m}^{2} \times \mathrm{g} / \mathrm{L} ; \text { this } \mathrm{mBMI} \\
\text { is mean of } 20 \\
\text { participants }\end{array}$ & $\begin{array}{l}\mathrm{mBMI}= \\
978 \\
(195) \\
\mathrm{kg} / \mathrm{m}^{2} \mathrm{x} \\
\mathrm{g} / \mathrm{L}\end{array}$ & $\begin{array}{l}\mathrm{BMI}= \\
20.9 \\
(3.1) \\
\mathrm{kg} / \mathrm{m}^{2}\end{array}$ \\
\hline
\end{tabular}

TABLE 2: Characteristics of participants of the studies included in the systematic review

SD: standard deviation; ATTR-ACT: Tafamidis in Transthyretin Cardiomyopathy Clinical Trial; * signifies median value (range); NA: not available; BMI: body mass index; TTR: transthyretin 


\section{Cureus}

\begin{tabular}{|c|c|c|c|c|c|c|}
\hline $\begin{array}{l}\text { Clinical Study } \\
\text { Characteristics }\end{array}$ & $\begin{array}{l}\text { ATTR-ACT trial } \\
\text { [12] }\end{array}$ & Maurer et al. [13] & $\begin{array}{l}\text { Damy et al. } \\
\text { [14] }\end{array}$ & Merlini et al. [15] & Cortese et al. [16] & Yukio et al. [17] \\
\hline $\begin{array}{l}\text { Study design; } \\
\text { Phase }\end{array}$ & $\begin{array}{l}\text { Multicenter, } \\
\text { double-blinded, } \\
\text { placebo- } \\
\text { controlled, } \\
\text { parallel design, } \\
\text { randomized; } \\
\text { Phase III }\end{array}$ & $\begin{array}{l}\text { Open level, single } \\
\text { treatment arm; Phase } \\
\text { ॥ }\end{array}$ & $\begin{array}{l}\text { Open level; } \\
\text { Phase II }\end{array}$ & $\begin{array}{l}\text { Open level, single } \\
\text { treatment arm; Phase II }\end{array}$ & $\begin{array}{l}\text { Multicenter observational } \\
\text { study }\end{array}$ & $\begin{array}{l}\text { Multicenter, single- } \\
\text { arm, open-label; } \\
\text { Phase III study }\end{array}$ \\
\hline Participants No. & 441 & 31 & 21 & 21 & 61 & 10 \\
\hline $\begin{array}{l}\text { Duration of } \\
\text { study }\end{array}$ & 30 months & 12 months & 12 months & 12 months & 36 months & $\begin{array}{l}30 \text { months (median } \\
\text { treatment duration } \\
713.5 \text { days) }\end{array}$ \\
\hline $\begin{array}{l}\text { Treatment } \\
\text { received }\end{array}$ & $\begin{array}{l}\text { Participants } \\
\text { received } \\
\text { Tafamidis } 80 \\
\mathrm{mg} \text {, Tafamidis } \\
20 \mathrm{mg} \text {, or } \\
\text { matching } \\
\text { placebo once } \\
\text { daily assigned in } \\
\text { the ratio of 2:1:2 }\end{array}$ & $\begin{array}{l}20 \mathrm{mg} \text { Tafamidis once } \\
\text { daily }\end{array}$ & $\begin{array}{l}20 \mathrm{mg} \\
\text { Tafamidis once } \\
\text { daily (in a soft } \\
\text { gelatin capsule) }\end{array}$ & $20 \mathrm{mg}$ Tafamidis once daily & $20 \mathrm{mg}$ Tafamidis once daily & $\begin{array}{l}\text { Once-daily oral } \\
\text { dose of Tafamidis } \\
\text { meglumine } 20 \mathrm{mg}\end{array}$ \\
\hline Control group & Placebo & None & None & None & None & None \\
\hline $\begin{array}{l}\text { Study } \\
\text { Population }\end{array}$ & $\begin{array}{l}\text { Transthyretin } \\
\text { amyloid } \\
\text { cardiomyopathy } \\
\text { (ATTRwt or } \\
\text { ATTRm) } \\
\text { confirmed } \\
\text { patients; } 18 \text { to } \\
90 \text { years }\end{array}$ & $\begin{array}{l}\text { Men and } \\
\text { postmenopausal } \\
\text { women } \geq 40 \text { years } \\
\text { with } \mathrm{V} 122 \text { or wild- } \\
\text { type transthyretin } \\
\text { cardiomyopathy } \\
\text { diagnosed via cardiac } \\
\text { biopsy or noncardiac } \\
\text { biopsy }\end{array}$ & $\begin{array}{l}\text { Symptomatic, } \\
\text { biopsy- } \\
\text { confirmed TTR } \\
\text { amyloid } \\
\text { polyneuropathy; } \\
18 \text { to } 75 \text { years; } \\
\text { men and non- } \\
\text { pregnant } \\
\text { women }\end{array}$ & $\begin{array}{l}\text { Symptomatic, biopsy- } \\
\text { confirmed TTR amyloid } \\
\text { polyneuropathy due to a } \\
\text { known pathogenic TTR } \\
\text { mutation other than } \\
\text { Val30Met or Val122lle; } 18 \\
\text { to } 75 \text { years; men and non- } \\
\text { pregnant women }\end{array}$ & $\begin{array}{l}\text { Women and men aged } \geq 18 \\
\text { affected by symptomatic } \\
\text { ATTR-related neuropathy; } \\
\text { patients starting treatment with } \\
\text { Tafamidis meglumine ( } 20 \mathrm{mg} \\
\text { once daily); written informed } \\
\text { consent to participate in the } \\
\text { study }\end{array}$ & $\begin{array}{l}\text { Men and women } \\
\text { aged } 20 \text { to } 75 \text { years } \\
\text { with documented } \\
\text { amyloid deposition } \\
\text { by biopsy and } \\
\text { Val30Met or other } \\
\text { TTR mutation (non- } \\
\text { Val30Met) }\end{array}$ \\
\hline
\end{tabular}

\section{TABLE 3: Study characteristics of the RCT and non-RCTs included in the review}

ATTR-ACT: Tafamidis in Transthyretin Cardiomyopathy Clinical Trial; ATTRwt: wild-type transthyretin-associated forms of amyloid; ATTRm: familial transthyretin-associated forms of amyloid; TTR: transthyretin; RCT: randomized controlled trial

\begin{tabular}{|c|c|c|c|c|c|c|}
\hline Results & ATTR-ACT trial [12] & Maurer et al. [13] & Damy et al. [14] & Merlini et al. [15] & $\begin{array}{l}\text { Cortese et al. } \\
{[16]}\end{array}$ & Yukio et al. [17] \\
\hline $\begin{array}{l}\% \text { of TTR } \\
\text { stabilization } \\
\text { achieved }\end{array}$ & NA & $\begin{array}{l}96.8 \% \text { and } 89.3 \% \\
\text { achieved TTR stabilization } \\
\text { after } 6 \text { weeks and } 12 \\
\text { months respectively. }\end{array}$ & 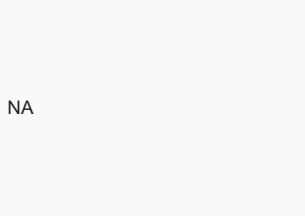 & $\begin{array}{l}100 \% \text { TTR } \\
\text { stabilization at } 6 \\
\text { and } 12 \text { months }\end{array}$ & NA & $\begin{array}{l}100 \% \text { TTR } \\
\text { stabilization at } \\
\text { week } 8 \text {, and } 26 \\
\text { whereas } 90 \% \\
\text { and } 80 \% \text { on } 52 \\
\text { and } 78 \text { weeks }\end{array}$ \\
\hline $\begin{array}{l}\text { Changes in } \\
\text { Echocardiographic }\end{array}$ & $\begin{array}{l}\text { The difference of change in \% } \\
\text { of circumferential and radial } \\
\text { mid-global strain from baseline } \\
\text { to month } 30 \text { between Tafamidis } \\
\text { and placebo group was } \\
\text { statistically significant; other } \\
\text { parameters like left ventricular }\end{array}$ & $\begin{array}{l}\text { Wall thickness and } \\
\text { pressure has no clinically }\end{array}$ & $\begin{array}{l}12 / 19 \text { Patients has new-onset } \\
\text { echocardiographic abnormalities; } 2 \\
\text { patients with normal IVS at } \\
\text { baseline, LV wall thickness } \\
\text { remained below > } 12 \mathrm{~mm} ; 4 / 12 \\
\text { patients demonstrated } \geq 2 \mathrm{~mm} \\
\text { increase in IVS thickness; } 6 \text { had } \\
\text { more than } 10 \% \text { increase in LV }\end{array}$ & $\begin{array}{l}\text { Echocardiographic } \\
\text { parameters } \\
\text { remained } \\
\text { relatively stable }\end{array}$ & $\begin{array}{l}\text { In } 34 \text { patients with } \\
\text { cardiac } \\
\text { involvement at } \\
\text { baseline Mean } \\
\text { IVS increased } \\
\text { from baseline by } \\
0.6 \pm 1.6 \mathrm{~mm} \text { at } \\
\text { M12 and } 1.05 \pm\end{array}$ & $\begin{array}{l}\text { Mean (SD) } \\
\text { change in } \\
\text { septum diastole } \\
\text { thickness from } \\
\text { baseline was } \\
\text { decreased by } \\
-1.3(\mathrm{SD}=2.7) \\
\mathrm{mm} \text { at Week } 52 \\
\text { and }-3.7 \\
(\mathrm{SD}=3.7) \mathrm{mm} \text { at }\end{array}$ \\
\hline
\end{tabular}




\section{Cureus}

$\begin{array}{ll}\text { parameters during } & \text { end-diastolic interventricular } \\ \text { the study period } & \text { septal wall thickness, left } \\ & \text { ventricular posterior wall } \\ & \text { thickness, left ventricular } \\ & \text { ejection fraction did not achieve } \\ & \text { statistical significance. }\end{array}$

Changes in the level of cardiac biomarkers during the study period rate due to adverse events was less in Tafamidis group; Common adverse events were blood and lymphatic disorders, cardiac disorders (cardiac failure and atrial fibrillation), eye disorders, gastrointestinal disorders (nausea, diarrhea, constipation

Lower in Tafamidis vs placebo

All-cause mortality (49.9\%); $\mathrm{HR}=0.75,95 \% \mathrm{Cl}=$ 0.51 to 0.96 ]

The frequency of cardiovascular-related

\section{Cardiovascular}

related

hospitalization

to placebo $[0.48$ per year vs [78/264 (29.5\%) vs 76/177

\section{hospitalizations was lower in the 7 patients were}

Tafamidis group in comparison hospitalized because of

cardiovascular events

NA

0.70 per year; relative risk ratio

of $0.68,95 \% \mathrm{Cl}=0.56$ to 0.81 ]

$\begin{array}{ll}\text { relevant changes seen on } & \text { mass; } 7 \text { has stable LV mass; } 1 \\ \text { the echocardiogram } & \text { patient has more than } 10 \% \\ & \text { decreased; none developed LV } \\ & \text { systolic dysfunction; LVEF } \\ & \text { deteriorated by more than } 10 \% \text { in } \\ & 2 / 18 \text { patients; no significant } \\ & \text { changes in left ventricular filling } \\ & \text { pressure }\end{array}$

from baseline to
month 12 with no
clinically relevant
changes

$2.0 \mathrm{~mm}$ at
M24; $15 \%$ of
patients showed
echocardiographic
evidence of
cardiac disease
progression

the end of the

study; the mean

increase in

stroke volume

was 1.1

$(\mathrm{SD}=5.0) \mathrm{mL}$ at

week 52 and 6.0

$(\mathrm{SD}=13.0) \mathrm{mL}$ at

the end of the

study

Baseline troponin I

concentration

remained stable

throughout the

study; the pro-

BNP level

remained

No clinically relevant changes in

increased with no

clinically relevant

changes over time

(mean increase of

pro-BNP

concentration

228.4 at month 6

and 306.6 at

month 12)

\section{Patients had \\ NT-proBNP NA \\ NA}

increase by $\geq 30 \%$
(1)
All 31 patients had $\geq 1$ AE; SAEs in 13/31; Cardiac failure in $8 / 31$; Atrial fibrillation in $3 / 31$ Fall in $3 / 31$; Syncope in 2/31

2 patients died during the study period: 1 patient resulting from $A L$ amyloidosis and the other died of hemorrhagic shock after a fall
3 discontinued early ( one due to TIA and 2 to undergo liver transplant); no life-threatening AEs or deaths; 8 patients had serious treatment-emergent $\mathrm{AEs}, 5$ experienced fall; 3 experienced one serious cardiovascular adverse event ( 1 coronary artery stenosis, one TIA, and $1 \mathrm{AV}$ block
$81 \%$ of

participants experienced at least one $\mathrm{AE}$, most common was falls; 4 SAES occurred that were considered related to treatment
85 all-cause $A E s$ were reported in 10 patients and $2 \mathrm{AEs}$ in 2 patients were treated as treatmentrelated AEs; severe AEs occurred in 3 patients pacemaker

implantation after

18 months of

treatment 


\section{Cureus}

\begin{tabular}{|c|c|c|c|c|c|c|}
\hline & & & & & & Tafamidis \\
\hline $\begin{array}{l}\text { Quality of Life } \\
\text { Assessments } \\
\text { during the study } \\
\text { duration }\end{array}$ & $\begin{array}{l}\text { Lower rate of decline in the } \\
\text { distance for the } 6 \text {-minute walk } \\
\text { test }(P<0.001) \text { and } K C C Q-O S \\
\text { score }(P<0.001) \text {. }\end{array}$ & $\begin{array}{l}\text { 6-minute walk test: the } \\
\text { mean distance walked } \\
\text { during 6-minute was } \\
\text { decreased by } 8.9 \text { meters } \\
\text { from baseline to month } 12 \text {. } \\
\text { Several measures of } \\
\text { health-related quality of } \\
\text { life: NHYA classification } \\
\text { maintained in } 71.4 \% \text { and } \\
\text { no patient deteriorated by } \\
\geq 2 \text { class or dropped to } \\
\text { NHYA classification IV; } \\
\text { overall patients } \\
\text { demonstrated a preserved } \\
\text { health-related quality of life }\end{array}$ & NA & $\begin{array}{l}\text { Mean changes in } \\
\text { TQOL score were } \\
-4.3 \text { at month } 6 \\
\text { and } 0.1 \text { at month } \\
12 \text {. }\end{array}$ & 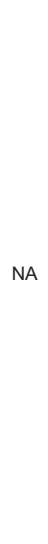 & $\begin{array}{l}\text { Mean change in } \\
\text { TQOL score } \\
\text { assessed by } \\
\text { Norfolk QOL-DN } \\
\text { from baseline } \\
\text { was } 11.8 \text { at } \\
\text { week } 26,9.1 \text { at } \\
\text { week } 52 \text {, and } \\
10.8 \text { at week } 78\end{array}$ \\
\hline
\end{tabular}

TABLE 4: Changes in echocardiographic parameters, cardiac biomarkers, quality of life, and \% of transthyretin stabilization achieved during the study

TTR: transthyretin; ATTR-ACT: Tafamidis in Transthyretin Cardiomyopathy Clinical Trial; NA: not available; IVS: interventricular septal thickness; LV: left ventricle; LVEF: left ventricular ejection fraction; M: month; SD: standard deviation; NT-proBNP: N-terminal pro-B-type natriuretic peptide; AE: Adverse Event; SAEs: serious adverse events; TIA: transient ischemic Attack; AV: Atrioventricular; HR: hazard ratio; Cl: confidence interval; AL: amyloid lightchain; NYHA: New York Heart Association; KCCQ-OS: Kansas city cardiomyopathy questionnaire-overall summary; TQOL: total quality of life; Norfolk QOL-DN: Norfolk quality of life-diabetic neuropathy questionnaire

TTR Stabilization

TTR stabilization was assessed in three non-RCTs [13,15,17], which compared baseline data with data at the end of the study. In Merlini et al. [15], 100\% of participants demonstrated TTR stabilization throughout the study period. At week 8 and week 26, TTR stabilization was maintained in $100 \%$ of participants in the Yukio et al. [17], but at weeks 52 and 78, it was maintained in $90 \%$ and $80 \%$ participants, respectively. In Yukio et al. [17], two participants had missing data regarding TTR stabilization at week 72. In the Maurer et al. [13], $96.8 \%$ of participants with wild-type TTR-CM had TTR stabilization at week 6 , whereas only $89.3 \%$ of participants maintained TTR stabilization at month 12. A randomized double-blinded trial that studied Tafamidis in TTR-FAP patients demonstrated TTR stabilization in $98 \%$ of patients in the Tafamidis group while none of the participants in the control group achieved TTR stabilization during the 18-month study period [18]. This explains the TTR stabilizing property of Tafamidis.

\section{Echocardiographic Changes}

Changes in findings of echocardiography were assessed in the ATTR-ACT trial [12] and five non-RCTs [1317]. In three studies (Maurer et al. [13], Damy et al. [14], and Merlini et al. [15]), there were no clinically relevant changes from baseline. Damy et al. [14] showed the occurrence of new-onset echocardiographic findings in 12/19 patients while Cortese et al. [16] demonstrated a 15\% progression of cardiac disease based on echocardiography. There were variable findings regarding interventricular septal (IVS) thickness.

Cardiac Biomarkers

Cardiac biomarkers were assessed in the ATTR-ACT trial [12] and four non-RCT studies [13-16]. NT-proBNP (N-terminal pro-B-type natriuretic peptide) level was evaluated in the ATTR-ACT trial [12] and four nonRCT studies [13-16], whereas Troponin I was evaluated in three non-RCT studies [13-15]. The NT-proBNP level was found to be stable in two studies (Damy et al. [14] and Merlini et al. [15]) while Cortese et al. [16] demonstrated an increase in NT-proBNP levels while in Maurer et al. [13], the increase was insignificant. Out of three studies evaluating Troponin I levels, the levels remained stable in two studies [12-13] and were found to be increased in one study [13].

Safety

The safety profile of Tafamidis was evaluated in the ATTR-ACT trial [12] and five non-RCTs [13-17]. In the ATTR-ACT trial, there were no significant differences in adverse events in the Tafamidis and placebo groups. All adverse events that appeared during the treatment period were mild to moderate in severity, and withdrawal of treatment due to adverse events was less in the Tafamidis group in comparison to placebo. In Maurer et al. [13], all patients with wild-type TTR-CM experienced at least one adverse event. Most of them 
had symptoms of heart failure while $22.6 \%$ (7/31) of participants experienced diarrhea and $16.1 \%$ (5/31) had weight gain. Serious adverse events occurred in $41.9 \%$ of participants (13/31), which included heart failure, atrial fibrillation, syncope, and pacemaker insertion. In the Maurer et al. study [13], two patients died and seven patients were hospitalized for cardiovascular events (CVEs) during the treatment period. In Damy et al. [14], the overall safety profile of Tafamidis was good. Only 38.1\% (8/21) of patients developed serious adverse events. In Merlini et al. [15], 81\% of participants (17/21) developed at least one adverse event during the study period. However, in Merlini et al. [15], only four patients had serious adverse events and no mortality was reported during the study period. In Cortese et al. [16], $13 \%$ of patients (8/61) experienced adverse events, of which three had serious adverse events. In the Yukio et al. [17] study, all 10 patients experienced at least one adverse event, but serious adverse events occurred only in three patients.

\section{All-Cause Mortality and Cardiovascular Related Hospitalization}

All-cause mortality and cardiovascular-related hospitalization were assessed in one RCT [12], which demonstrated a decrease in all-cause mortality in patients under Tafamidis vs. placebo. Maurer et al. [13] demonstrated minimal deaths and hospitalization in patients treated with Tafamidis. Cortese et al. [16] showed the progression of 12 patients to a higher NYHA (New York Heart Association) class, and one patient required pacemaker implantation.

Quality of Life

Quality of life was assessed in the ATTR-ACT trial [12] and three non-RCTs (Maurer et al. [13], Merlini et al. [15], and Yukio et al. [17]). The ATTR-ACT trial [12] and Maurer et al. [13] showed a lower rate of decline in the six-minute walk test. Yukio et al. [17] and Merlini et al. [15] demonstrated that quality of life was maintained throughout treatment with Tafamidis. Yukio et al. [17] also showed maintenance of ambulatory status in $50 \%$ of patients during their treatment.

\section{Modified BMI}

Modified BMI (mBMI) was assessed in three non-RCTs (Merlini et al. [15], Cortese et al. [16], and Yukio et al. [17]) where no changes were seen in Cortese et al. [16], and modified BMI increase was demonstrated in Yukio et al. [17]. Merlini et al. [15] showed an initial decrease in BMI followed by an increase in modified BMI.

\section{Discussion}

TTR tetramer dissociation has been the rate-limiting step in TTR amyloid formation. Tafamidis is one of the TTR stabilizing compounds that have been studied well. It is essential to give evidence to the scientific community relating the different effects of Tafamidis on clinical parameters. This will help establish the role of Tafamidis in transthyretin amyloid cardiomyopathy.

In Maurer et al. [13], 30 participants with wild-type TTR-CM had increased interventricular septum and left ventricular posterior wall thickness. Out of 30 wild TTR-CM patients in the Maurer et al. study [13], 73.7\% of patients had elevated left ventricular filling pressure while $64 \%$ of participants had elevated right ventricular filling pressure, and $43.3 \%$ had an ejection fraction of $<50 \%$. In the Maurer et al. study [13], throughout the treatment period with Tafamidis, 30 patients with wild-type TTR-CM did not have clinically relevant changes in the above parameters. In Damy et al. [14], participants had several echocardiographic abnormalities at baseline like LV posterior wall thickness, interventricular septal thickness, and isovolumetric relaxation time. However, during the 12-month treatment period with Tafamidis, the average values for these echocardiographic parameters remained stable. In Merlini et al. [15], echocardiographic parameters like posterior LV wall thickness, interventricular septal thickness, left atrial diameter, left ventricular mass, and LV ejection fraction were assessed at baseline and month 12 of the study. During the treatment period, participants of Merlini et al. [15] had relatively stable echocardiographic parameters over 12 months of treatment with Tafamidis without any clinically significant changes. In Cortese et al. [16], 34 patients out of 61 had cardiac involvement, of which mean interventricular septum thickness increased by $0.6 \pm 1.6 \mathrm{~mm}$ at month 12 and $1.05 \pm 2 \mathrm{~mm}$ at month 24 but only five patients had cardiac disease progression. During the study duration of Cortese et al. [16], out of 23 patients without cardiac involvement, eight developed cardiomyopathy, and 12 patients progressed to a higher NYHA heart failure class. In Yukio et al. [17], interventricular septum diastolic thickness decreased at the end of the study while stroke volume increased at the end of the study.

Measurement of myocardial strain is a method to quantify left ventricular function by using speckle-tracking echocardiography. There has been a system to measure strain along three cardiac axes: circumferential, radial, and longitudinal [19]. The three-axis system will allow measuring the shortening and elongation of the myocardium through the cardiac cycle in three directions in reference to the measurements during the time of QRS-complex [19]. Among the measurements in three cardiac axes, global longitudinal strain (GLS) is more sensitive and important for the measurement of systolic function than left ventricular ejection fraction [19]. GLS has the power to uncover even subtle or subclinical LV dysfunction in cardiomyopathies. In 
the ATTR-ACT trial [12], the difference of change in percentage of circumferential and radial mid-global strain from baseline to month 30 between the Tafamidis and placebo groups was statistically significant. However, the difference of change in percentage of global longitudinal strain from baseline to month 30 between the Tafamidis and placebo groups was not statistically significant. Another echocardiographic parameter that gained statistical significance was the difference in change in volume of left ventricular stroke volume from baseline to month 30 between the Tafamidis and placebo groups (LS mean difference $6.28 \mathrm{ml}$; $95 \%$ CI 1.96 to 10.59). The decrease in left ventricular stroke volume was less (5.38 ml) in the Tafamidis group in comparison to the placebo $(11.66 \mathrm{ml})$. Also, other parameters like left ventricular enddiastolic interventricular septal wall thickness, left ventricular posterior wall thickness, and left ventricular ejection fraction did not achieve statistical significance.

Therefore, there was the stability of different echocardiographic parameters in single-arm non-RCT studies. In the ATTR-ACT trial [12], the scenario is different. Although there was a statistically significant difference in the change in stroke volume at the end of the trial between the Tafamidis and placebo groups, with a lower decrease in the Tafamidis group, there was no statistically significant difference of changes noted in other echocardiographic parameters during the follow-up period.

In Damy et al. [14], 76.2\% of patients had ECG abnormalities at baseline. During the study period of 12 months, $42.9 \%$ of patients developed new-onset ECG abnormalities o,f which arrhythmias were most common. In the Maurer et al. [13] study, 100\% of participants had ECG abnormalities while $80 \%$ of participants had abnormalities during Holter monitoring. During the study period, ECG abnormalities associated with treatment were low. In Merlini et al. [15], 44.4\% of participants developed a treatmentassociated ECG abnormality. In patients with amyloidosis, ECG and Holter abnormalities represent cardiac autonomic dysfunction [14]. In the above studies, we can find treatment-associated ECG abnormalities to be mild to moderate. Further, the ECG and Holter abnormalities that existed at baseline could be co-related with a cardiac abnormality at baseline. However, to prove that Tafamidis improves and prevents cardiac autonomic dysfunction, a large prospective cohort study and a randomized clinical trial are needed.

NT-proBNP indicates raised intraventricular pressures in congestive heart failure and carries a significant prognostic value for mortality and cardiac disease progression [20]. Also, some evidence suggests NTproBNP be an important marker of cardiac hypertrophy and LV function in hereditary TTR amyloidosis patients [21-22]. In the Maurer et al. [13] study, the median NT-proBNP of participants was raised. Although there were many variations in NT-proBNP values among participants, there was no significant increase in NT-proBNP at the end of the study in comparison to baseline. In the Damy et al. [14] and Merlini et al. [15] studies, mean NT-proBNP was raised at the start of the study, but no significant elevation was noted during the study period with Tafamidis. In the Cortese et al. study [16], 42\% of patients had altered NT-proBNP level, but during the treatment period with Tafamidis, only three of 23 patients without heart involvement had an increase of NT-proBNP by $30 \%$. In the ATTR-ACT trial [12], while exploring other endpoints, we found a statistically significant smaller increase in the NT-proBNP level among participants of the Tafamidis group than placebo at months 12 and 30. This is in contrast to non-RCT studies that show either a decrease or stable NT-proBNP level among the Tafamidis group. Hence, there are variations in the change in the level of NT-proBNP marker between single-arm non-RCTs and RCT.

In the Damy et al. [14] and Merlini et al. [15] studies, the mean Troponin I level of participants was normal before the start of the study and remained stable during the study period. This is consistent with a previous study that reports NT-proBNP as a more sensitive marker of cardiomyopathy in patients with TTR amyloidosis than Troponin I [21]. The stability in the value of Troponin I during the study period probably could be attributed to Tafamidis in halting the progression of cardiac disease in TTR-CM patients. In the Maurer et al. [13] study, 100\% of participants had raised Troponin I at baseline. Following an initial drop in the concentration of Troponin I at month 3, Troponin I level increased at month 12 in comparison to baseline. However, the increase in Troponin I was comparable to the TRACS (Transthyretin Amyloidosis Cardiac Study) study where a group of untreated TTR-CM patients had a similar elevation in Troponin I [23].

ATTR-ACT [12] is a placebo-controlled randomized multicenter trial that studied the efficacy of Tafamidis in transthyretin amyloid cardiomyopathy patients. In the ATTR-ACT trial [12], the all-cause mortality was lower in the Tafamidis group in comparison to placebo [HR=0.70, 95\% CI, 0.51 to 0.96]. Previous case series have reported a one-year mortality rate of $14 \%$ to $23 \%$ in wild-type TTR-CM patients. Hence, the reduction in all-cause mortality associated with Tafamidis is significant when we consider the natural history of the disease. Also, in the ATTR-ACT trial [12], the Tafamidis group had a lower rate of cardiovascular-related hospitalizations in comparison to placebo (relative risk ratio $=0.68$; $95 \% \mathrm{CI}, 0.56$ to 0.81 ).

In the ATTR-ACT trial, the distance covered during the six-minute walk test was prevented from further deteriorating. The Kansas City Cardiomyopathy Questionnaire-Overall Summary (KCCQ-OS) scoring system is the measure of functional capacity and quality of life in cardiomyopathy patients. Scores in the lower range in the KCCQ-OS scoring system denote a low quality of life. In the ATTR-ACT trial [12], Tafamidis slowed the lowering of the KCCQ-OS score. In the Maurer et al. [13] study, most of the patients maintained the distance achieved during the six-min walk test. Also, in Maurer et al. [13], 71.4\% of patients had stable NYHA classification with no patient deteriorating two classes or reaching the IV NYHA classification. In Maurer et al. [13], the overall quality of life of participants was maintained on different scoring systems like 
global patient assessment, Kansas City Cardiomyopathy Questionnaire, and Short Form 36 scoring systems. In the Merlini et al. [15] study, Norfolk Quality of life-Diabetic Neuropathy was used to assess the quality of life where higher scores denote a worse quality of life. The quality of life scoring was maintained during the study period in Merlini et al. [15] study. In the Yukio et al. [17] study, during the treatment duration of 1.5 years, walking was preserved in $50 \%$ of patients. Also, in the Yukio et al. [17] study, change in the total quality of life from baseline was stable at weeks 26,52 , and 78 .

Hence, considering the findings of non-RCTs, we can associate Tafamidis with reducing cardiac disease progression in patients with TTR-CM. Analyzing the echocardiographic parameters, cardiac biomarkers (NTProBNP, Troponin I), ECG, and quality of life measures in five non-RCTs, the measurement parameters were stable during the treatment period. But in the absence of a control group in five non-RCTs, it is challenging to predict the changes in the control group. However, the change in parameters being stable in all five nonRCTs rules out the findings to be due to chance alone. In the TRACS study, 18 wild-type and 11 V122I patients with TTR-CM were followed without treatment for 12 months. In the TRACS study, the ejection fraction of participants as measured by echocardiography decreased by $-8 \%$ to $50.5 \%$, whereas the NTproBNP level was raised by 1487 to $6268 \mathrm{pg} / \mathrm{ml}$. Therefore, correlating the findings of the TRACS study [23] with the findings of five non-RCTs, we can positively associate Tafamidis use and stable cardiac parameters during the study period. Also, we can say the drug has a good safety profile by analyzing the safety profile of Tafamidis in the ATTR-ACT trial [12] and five non-RCTs [13-17].

Thus, the ATTR-ACT trial [12] shows a decrease in all-cause mortality and cardiovascular-related hospitalizations in the Tafamidis group in comparison to placebo. But assessing the progression of disease in terms of cardiac parameters, we get variances in findings in the ATTR-ACT trial in comparison to singlearm non-RCTs included in this review. Hence, further randomized clinical trials that study different echocardiographic parameters are needed to provide robust evidence of Tafamidis in reducing cardiac disease progression. Currently, there are few ongoing clinical trials of Tafamidis. NCT04814186 is a phase 4 clinical trial of Tafamidis registered with ClinicalTrials.gov on March 24, 2021, to evaluate the safety and efficacy of once-daily treatment in Chinese participants with TTR-CM, which is expected to be completed by November 24, 2023. NCT02791230 is an ongoing phase 3 clinical trial registered with ClinicalTrials.gov on June 6, 2016, to study the safety of once-daily treatment with Tafamidis meglumine in TTR-CM patients. The findings of these clinical trials will further provide additional evidence of the safety and efficacy of Tafamidis in TTR-CM patients.

\section{Conclusions}

Tafamidis is a TTR-stabilizing drug that has been associated with reducing cardiovascular events and allcause mortality in patients with TTR-CM disease. Analyzing the non-RCTs findings, we can associate the drug with stabilizing cardiac parameters and halting the cardiac disease progression. However, analyzing the echocardiographic parameters and biomarkers in the ATTR-ACT trial, we get variances in findings in comparison to single-arm non-RCTs. On overall analysis, we can say for sure that Tafamidis is a safe and effective drug for TTR-CM patients. However, the results of future RCTs of Tafamidis can provide robust evidence on the role of Tafamidis in halting cardiac disease progression in transthyretin amyloid cardiomyopathy.

\section{Appendices}

\section{Search strategy}

PubMed: 87

((("amyloid cardiomyopath*") OR ("amyloid cardiomyopath*"[Title/Abstract])) OR ("amyloid cardiomyopath*"[MeSH Terms]) OR ("Cardiac amyloid*")) OR (("Cardiac amyloid*")[Title/Abstract])) OR (("Cardiac amyloid*")[MeSH Terms])

PubMed Central (PMC): 370

((("tafamidis"[Supplementary Concept] OR "tafamidis"[All Fields]) OR Tafamidis[Title]) OR Tafamidis[Abstract]) AND ((("amyloid cardiomyopathy"[All Fields] OR "amyloid cardiomyopathy*" [Abstract]) OR "amyloid cardiomyopathy*"[Title]) OR (("cardiac amyloid*"[All Fields] OR "cardiac amyloid*"[Abstract]) OR "cardiac amyloid*"[Title]))

Cochrane Library: 42

Date Run: 06/27/2021

Comment: 
Google Scholar: 192

Tafamidis AND ("amyloid cardiomyopathy" OR "cardiac amyloidosis")

Total no. of articles: 691

\section{Additional Information \\ Disclosures}

Conflicts of interest: In compliance with the ICMJE uniform disclosure form, all authors declare the following: Payment/services info: All authors have declared that no financial support was received from any organization for the submitted work. Financial relationships: All authors have declared that they have no financial relationships at present or within the previous three years with any organizations that might have an interest in the submitted work. Other relationships: All authors have declared that there are no other relationships or activities that could appear to have influenced the submitted work.

\section{References}

1. Westermark P, Benson MD, Buxbaum JN, et al.: A primer of amyloid nomenclature. Amyloid. 2007, 14:17983. 10.1080/13506120701460923

2. Falk RH, Alexander KM, Liao R, Dorbala S: AL (light-chain) cardiac amyloidosis: a review of diagnosis and therapy. J Am Coll Cardiol. 2016, 68:1323-41. 10.1016/j.jacc.2016.06.053

3. Molina O G, Judge D, Campbell W, Chahal H, Mugmon M: Transthyretin cardiac amyloidosis: an underdiagnosed cause of heart failure. J Community Hosp Intern Med Perspect. 2014, 4:25500. 10.3402/jchimp.v4.25500

4. Grogan M, Scott CG, Kyle RA, et al.: Natural history of wild-type transthyretin cardiac amyloidosis and risk stratification using a novel staging system. J Am Coll Cardiol. 2016, 68:1014-20. 10.1016/j.jacc.2016.06.033

5. Vollmar J, Schmid JC, Hoppe-Lotichius M, et al.: Progression of transthyretin (TTR) amyloidosis in donors and recipients after domino liver transplantation-a prospective single-center cohort study. Transpl Int. 2018, 31:1207-15. 10.1111/tri.13326

6. Sousa M, Monohan G, Rajagopalan N, Grigorian A, Guglin M: Heart transplantation in cardiac amyloidosis. Heart Fail Rev. 2017, 22:317-27. 10.1007/s10741-017-9601-z

7. Ruberg FL, Grogan M, Hanna M, Kelly JW, Maurer MS: Transthyretin amyloid cardiomyopathy: JACC stateof-the-art review. J Am Coll Cardiol. 2019, 73:2872-91. 10.1016/j.jacc.2019.04.003

8. Page MJ, McKenzie JE, Bossuyt PM, et al.: The PRISMA 2020 statement: an updated guideline for reporting systematic reviews. BMJ. 2021, 372:n71. 10.1136/bmj.n71

9. Moher D, Shamseer L, Clarke M, et al.: Preferred reporting items for systematic review and meta-analysis protocols (PRISMA-P) 2015 statement. Syst Rev. 2015, 4:1. 10.1186/2046-4053-4-1

10. Sterne JA, Savović J, Page MJ, et al.: RoB 2: a revised tool for assessing risk of bias in randomised trials . BMJ. 2019, 366:14898. 10.1136/bmj.14898

11. Downs SH, Black N: The feasibility of creating a checklist for the assessment of the methodological quality both of randomised and non-randomised studies of health care interventions. J Epidemiol Community Health. 1998, 52:377-84. 10.1136/jech.52.6.377

12. Maurer MS, Schwartz JH, Gundapaneni B, et al.: Tafamidis treatment for patients with transthyretin amyloid cardiomyopathy. N Engl J Med. 2018, 379:1007-16. 10.1056/NEJMoa1805689

13. Maurer MS, Grogan DR, Judge DP, et al.: Tafamidis in transthyretin amyloid cardiomyopathy. Effects on transthyretin stabilization and clinical outcomes. Circ Heart Fail. 2015, 8:519-26. 10.1161/CIRCHEARTFAILURE. 113.000890

14. Damy T, Judge DP, Kristen AV, Berthet K, Li H, Aarts J: Cardiac findings and events observed in an openlabel clinical trial of tafamidis in patients with non-Val30Met and non-Val122Ile hereditary transthyretin amyloidosis. J Cardiovasc Transl Res. 2015, 8:117-27. 10.1007/s12265-015-9613-9

15. Merlini G, Planté-Bordeneuve V, Judge DP, et al.: Effects of tafamidis on transthyretin stabilization and clinical outcomes in patients with non-Val30Met transthyretin amyloidosis. J Cardiovasc Transl Res. 2013, 6:1011-20. 10.1007/s12265-013-9512-x

16. Cortese A, Vita G, Luigetti M, et al.: Monitoring effectiveness and safety of Tafamidis in transthyretin amyloidosis in Italy: a longitudinal multicenter study in a non-endemic area. J Neurol. 2016, 263:916-24. 10.1007/s00415-016-8064-9

17. Ando Y, Sekijima Y, Obayashi K, et al.: Effects of tafamidis treatment on transthyretin (TTR) stabilization, efficacy, and safety in Japanese patients with familial amyloid polyneuropathy (TTR-FAP) with Val30Met and non-Val30Met: a phase III, open-label study. J Neurol Sci. 2016, 362:266-71. 10.1016/j.jns.2016.01.046

18. Coelho T, Maia LF, Martins da Silva A, et al.: Tafamidis for transthyretin familial amyloid polyneuropathy. A randomized, controlled trial. Neurology. 2012, 79:785-92. 10.1212/WNL.0b013e3182661eb1

19. Smiseth OA, Torp H, Opdahl A, Haugaa KH, Urheim S: Myocardial strain imaging: how useful is it in clinical decision making?. Eur Heart J. 2016, 37:1196-207. 10.1093/eurhearti/ehv529

20. Bhardwaj A, Rehman SU, Mohammed A, Baggish AL, Moore SA, Januzzi JL Jr: Design and methods of the 


\section{Cureus}

Pro-B Type Natriuretic Peptide Outpatient Tailored Chronic Heart Failure Therapy (PROTECT) study. Am Heart J. 2010, 159:532-538.e1. 10.1016/j.ahj.2010.01.005

21. Suhr OB, Anan I, Backman C, Karlsson A, Lindqvist P, Mörner S, Waldenström A: Do troponin and Bnatriuretic peptide detect cardiomyopathy in transthyretin amyloidosis?. J Intern Med. 2008, 263:294-301. 10.1111/j.1365-2796.2007.01888.x

22. Damy T, Deux JF, Moutereau S, et al.: Role of natriuretic peptide to predict cardiac abnormalities in patients with hereditary transthyretin amyloidosis. Amyloid. 2013, 20:212-20. 10.3109/13506129.2013.825240

23. Ruberg FL, Maurer MS, Judge DP, et al.: Prospective evaluation of the morbidity and mortality of wild-type and V122I mutant transthyretin amyloid cardiomyopathy: the Transthyretin Amyloidosis Cardiac Study (TRACS). Am Heart J. 2012, 164:222-228.e1. 10.1016/j.ahj.2012.04.015 